\title{
ON THE DYNAMIC ANALYSIS OF A CAM MECHANISM \\ WITH BEARING CLEARANCES
}

\author{
B. M. Bahgat \\ M.O.M.Osman ***
}

ABSTRACT

The paper develops a procedure for the dynamic analysis of a cam mechanism. with bearing clearances. The analysis mainly relies on determining the clearance angles $\beta_{i j}$ and its first and second derivatives. The governing equations of each clearance angle are developed on the condition that the variations in displacements and velocities due to clearance effect are very small and could be ignored. Also, the equations of these clearance angles: are developed such that the common normal at the point of contact at each clearance connection coincides with the direction of the clearance eccentricity vector which has the same direction of the normal reaction force at the point of contact. A straight sided cam operating a roller follower. is considered to illustrate the procedure.

\section{INTRODUCTION}

Bearing clearances due to manufacturing tolerances and subsequent wear have undesired effects on the dynamic behavior of machinery rotating at high : ispeed. Although, the problem has a complexity, there is a need for a systematic procedure to estimate quantitatively the accelerations and bearing. forces as affected by these bearing clearances.

- Chen $|1|^{* *}$ presented a method to obtain the dynamic response of the cam:driven systems numerically. Wiederrich and Roth $|2|$ presented methods for dynamic synthesis of cams using finite trigonometric series. Koster $|3|$ irvestigated the dynamic behavior of a follower driven by a flexible shaft. Chen and Polvanish |4-5|investigated the dynamic response of the cam-driven mechanism based on a linear and non-linear lumped system models of single: and double freedom. The dynamic synthesis of modeled cam systems with one and two degrees of freedom is reported by Matthew and Tesar $|6-8,10|$ to validate a number of fundamental design rules of thumb pertaining to classification, motion specifications, motion distortion etc. Koster |9| providẹd :a digital simulation program based on a model with four degrees of freedom.

The dynamic behavior of a cam-actuated system is reported by chen $|11|$, in :

* Associate Professor, Mechanical Design Department Cairo University Cairo, Egypt, A.R.E.

**Numbers in brackets | |designate references at end of paper

***Associate Professor, Machanical Design Department Cairo University

LCairo, Egypt,A.R.E. 
which the follower is coupled with a non-linear pneumatic mechanism of hysteric type and contains a compressible fluid on a plunger. Sankar and Osman $|12|$ investigated the dynamic accuracy in cam production using hybrid: profiling mechanism in turning with a single point cutting tool. The optimum elastodynamic synthesis of a cam-follower train is reported by Rao $|13|$, in which, he found the retainer spring constant (stiffness) and the mass of follower train required to give minimum flexibility error and also to prevent separation between cam and follower. Szakallas and Savage $|14|$ used dimensional analysis and found two ratios which characterize the level of vibration in the cam-driven system.

In this work, the dynamic analysis of a cam mechanism with bearing clear- : ances is carried out. The clearance angles $\beta_{i j}$ are obtained using three conditions as follows:

1) The variation in displacements due to clearance effects are negligible. 2) The variations in velocities due to clearance effects are negligible. and 3) The common normal at the point of contact coincides with the direc; : tion of the bearing eccentricity vector $\vec{\Delta}_{i j}$ and also coincides with the direction of the bearing reaction force at the point of contact.

: The estimated clearance angles together with its first and second derivatives are systematically used to evaluate kinematic and dynamic quantities of the cam mechanism.

A, straight sided cam operating a roller follower with two bearing clearances is used to illustrate the procedure.

\section{ANALYTICAL METHOD}

In this section a system of equations is developed which are valid for all: contact conditions at any cam rotational angle $\theta_{2}$ in the period of the cam action. A simultaneous contact at joints with clearance is assumed and used in the dynamic analysis of the cam mechanism. This is a reasonable assumption, since a separation at any joint will last only an infinitesmal small period of time $(\Delta t \rightarrow 0)$. Therefore, the solution during the separation period $(\Delta t>0)$ is obtained graphically by joining the result's of the two contact points before and after impact.

Figure 1 shows a straight sided cam operating a roller follower with two bearing clearances which is considered in this analysis. One, 2,3, and 4 are link numbers corresponding to the frame, cam, roller and follower respectively.

Referring to figures 1 and 2, the angles $\alpha$ and $\gamma$ and be expressed as,

$$
r=\cos ^{-1}\left[\left(r_{b}-r_{n}\right) / d\right]
$$

and $\quad \alpha=\tan ^{-1}\left[d \sin \gamma /\left(r+r_{b}\right)\right]$

Three cases $A, B$ and $C$ are considered through the analysis.

L 
.

Case A : Contact with Flate Flank During Upward Motion of the Follower

Figure 2 shows the contact at any instant during this period, where,

$$
(\pi / 2-\gamma) \leqslant \theta_{2} \leqslant(\pi / 2-\gamma+\alpha) \text {. }
$$

From figure 2, the distances $r_{i}$ and $q$ which is the projection of vector $\vec{D}$ : : along the $\mathrm{X}$ direction and their derivatives can be written as,

$$
\begin{aligned}
& r_{1}=\left(r+r_{b}\right)\left(1-\cot \gamma \tan \theta_{2}^{\prime}\right) \\
& \dot{r}_{1}=-\left(r+r_{b}\right) \omega_{2} \cot \gamma \sec ^{2} \theta_{2}^{\prime} \\
& \quad \ddot{r}_{1}=-2\left(r+r_{b}\right) \omega_{2}^{2} \cot \gamma \sec ^{2} \theta_{2}^{\prime} \tan \left(\theta_{2}^{\prime}\right. \\
& q=D \cos \theta_{2}=\Delta_{43} \cos \beta_{43}-r_{1} \cos \left(\gamma+\theta_{2}\right)-\Delta_{12} \cos \beta_{12} \\
& \dot{q}=-\Delta_{43} \dot{B}_{43} \sin B_{43}-\dot{r}_{1} \cos \left(\gamma+\theta_{2}\right)+r_{1} \omega_{2} \sin \left(\gamma+0_{2}\right)+\Lambda_{12} \dot{B}_{12} \sin B_{12} \vdots \\
& \ddot{q}=-\Delta_{43}\left(\ddot{\beta}_{43} \sin \beta_{43}+\ddot{\beta}_{43}^{2} \cos \beta_{43}\right)+\Delta_{12}\left(\ddot{\beta}_{12} \sin \beta_{12}+\dot{\beta}_{12}^{2} \cos \beta_{12}\right) \\
& -\left[\left(\ddot{r}_{1}-r_{1} \omega_{2}^{2}\right) \cos \left(\gamma+\theta_{2}\right)-2 \dot{r}_{1} \omega_{2} \sin \left(\gamma+\theta_{2}\right)\right]
\end{aligned}
$$

where

$$
\theta_{2}^{\prime}=\theta_{2}-\left(\frac{\pi}{2}-\gamma\right)
$$

The follower displacement, velocity and acceleration $\mathrm{Y}_{4}, \dot{Y}_{4}, \ddot{Y}_{4}$ can be expressed as

$$
\begin{aligned}
Y_{4}= & \Delta_{12} \sin \beta_{12}+q \tan \theta_{2}+r_{1} \sin \left(\gamma+\theta_{2}\right)-\Delta_{43} \sin \beta_{43}-\left(r+r_{b}\right) \\
\dot{Y}_{4}= & \Delta_{12} \dot{\beta}_{12} \cos \beta_{12}+\dot{q} \tan \theta_{2}+q \omega_{2} \sec ^{2} \theta_{2}+\dot{r}_{1} \sin \left(\gamma+\theta_{2}\right) \\
& +r_{1} \omega_{2} \cos \left(\gamma+\theta_{2}\right)-\Delta_{43} \dot{\beta}_{43} \cos \beta_{43} \\
\ddot{Y}_{4}= & \Delta_{12}\left(\ddot{\beta}_{12} \cos \beta_{12}-\dot{\beta}_{12}^{2} \sin \beta_{12}\right)-\Delta_{43}\left(\ddot{\beta}_{43} \cos \beta_{43}-\dot{\beta}_{43}^{2} \sin \beta_{43}\right) \\
& +\left(\ddot{q} \tan \theta_{2}+2 \dot{q}_{2} \sec ^{2} \theta_{2}+2 q \omega_{2}^{2} \sec ^{2} \theta_{2} \tan \theta_{2}\right) \\
& +\left[\left(\ddot{r}_{1}-r_{1} \omega_{2}^{2}\right) \sin \left(\gamma+\theta_{2}\right)+2 \dot{r}_{1} \omega_{2} \cos \left(\gamma+\theta_{2}\right)\right]
\end{aligned}
$$

¿.. 
Using D'Alembert principle, the dynamic equations of the cam assembly, roller and follower are given by,

$\vdots$

$$
\begin{aligned}
& R_{12}^{X}-R_{32} \cos \left(\gamma+\theta_{2}\right)=m_{2} \ddot{X}_{2} \\
& -R_{12}^{y}-R_{32} \sin \left(\gamma+\theta_{2}\right)=m_{2} \ddot{Y}_{2} \\
& -R_{43}^{X}-R_{32} \cos \left(\gamma+\theta_{2}\right)=m_{3} \ddot{X}_{3} \\
& R_{43}^{y}-R_{32} \sin \left(\gamma+\theta_{2}\right)=m_{3} \ddot{Y}_{3} \\
& m_{4} \ddot{Y}_{4}+C \dot{Y}_{4}+K Y_{4}=R_{43}^{y}-F_{0} \\
& T_{2}=m_{2} S\left(\ddot{Y}_{2} \cos \theta_{2}-\ddot{X}_{2} \sin \theta_{2}\right)+\left(q \sin \gamma / \cos \theta_{2}\right) R_{32}
\end{aligned}
$$

where:

$$
\begin{aligned}
& \ddot{x}_{2}=-\operatorname{s\omega } \omega_{2}^{2} \cos \theta_{2}-\Delta_{12}\left(\ddot{\beta}_{12} \sin \beta_{12}+\dot{\beta}_{12}^{2} \cos \beta_{12}\right) \\
& \ddot{y}_{2}=-\operatorname{s\omega } \omega_{2}^{2} \sin \theta_{2}+\Delta_{12}\left(\ddot{\beta}_{12} \cos \beta_{12}-\dot{\beta}_{12}^{2} \sin \beta_{12}\right) \\
& \ddot{x}_{3}=-\Delta_{43}\left(\ddot{\beta}_{43} \sin \beta_{43}+\dot{\beta}_{43}^{2} \cos \beta_{43}\right) \\
& \ddot{y}_{3}=\ddot{y}_{4}+\Delta_{43}\left(\ddot{\beta}_{43} \cos \beta_{43}-\dot{\beta}_{43}^{2} \sin \beta_{43}\right)
\end{aligned}
$$

Equation (8) are the same for the three cases of contact A, B and C. If the clearance angles $\left(\beta_{12}, \beta_{43}\right)$ and its derivatives $\left(\beta_{12}, \dot{\beta}_{43}\right),\left(\ddot{\beta}_{12}, \ddot{\beta}_{43}\right)$ are known, equations (2) to (8) can be used to evaluate displacement, $\rightarrow$ yelocity, acceleration of follower $\left(Y_{4}, \dot{Y}_{4}, \ddot{Y}_{4}\right)$, the dynamic reactions $\left(\vec{K}_{12}\right.$, $\vec{R}_{32}, \vec{R}_{43}$ ) and the torque $T_{2}$.

\section{Clearance Angles}

: The follower displacement $\mathrm{Y}_{4}$ is given by equation (6). From figure 2, the follower velocity $\dot{Y}_{4}$ can be written as,

$$
\dot{V}_{4}=\omega_{2} \cdot \overline{0_{12} O_{42}}
$$

where 
$$
\therefore \quad \sigma_{12} \sigma_{42}=-\left[\left(\Delta_{12} \sin \beta_{12}+q \tan \theta_{2}\right) / \tan \left(\gamma+\theta_{2}\right)\right]-r_{1} \cos \left(\gamma+\theta_{2}\right)
$$

$$
+\Delta_{43} \cos \beta_{43}
$$

Since, the variations of $\mathrm{Y}_{4}$ and $\dot{Y}_{4}$ due to clearance effect are negligible, : these conditions lead to,

$$
\begin{aligned}
& \therefore \quad f_{1}=\sin \left(\beta_{12}-\theta_{2}\right)-\Delta \sin \left(\beta_{43}-\theta_{2}\right)=0 \\
& \vdots \quad f_{2}=\sin \left(\beta_{12}-\theta_{2}\right)-\Delta f\left(\theta_{2}\right) \cos \beta_{43}=0
\end{aligned}
$$

: Where,

$\vdots$

$$
\Delta=\left|\vec{\Delta}_{43}\right| /\left|\vec{\Delta}_{12}\right|
$$

and $f\left(\theta_{2}\right)=-\sin \theta_{2}+\cos \theta_{2} \tan \left(\gamma+\theta_{2}\right)$

Equations (11) are solved for the clearance angles $\left(\beta_{12}, \beta_{43}\right)$ using Newton Raphson's method. The derivatives of equations (1l) give the angular ve: locities of clearance angles $\left(\dot{\beta}_{12}, \dot{\beta}_{43}\right)$ as,

$\vdots$

$$
\begin{aligned}
& \dot{\beta}_{43}=\omega_{2}\left[f^{\prime}\left(\theta_{2}\right) \cos \beta_{43}+\cos \left(\beta_{43}-\theta_{2}\right)\right] /\left[f\left(\theta_{2}\right) \sin \beta_{43}+\cos \left(\beta_{43}-\theta_{2}\right)\right] \\
& \dot{\beta}_{12}=\Delta \xi \dot{\beta}_{43}+(1-\Delta \xi) \omega_{2}
\end{aligned}
$$

: where,

$$
\xi=\cos \left(\beta_{43}-\theta_{2}\right) / \cos \left(\beta_{12}-\theta_{2}\right)
$$

and

$$
f^{\prime}\left(\theta_{2}\right)=-\cos \theta_{2}-\sin \theta_{2} \tan \left(\gamma+\theta_{2}\right)+\cos \theta_{2} \sec ^{2}\left(\gamma+\theta_{2}\right)
$$

$\vdots$ Equation (12) is used to evaluate $\left(\dot{\beta}_{12}, \dot{\beta}_{43}\right)$. Since the directions of the clearancef vectors $\vec{\Delta}_{12}$ and $\vec{\Delta}_{43}$ coincide with the directions of the re: actions $\vec{R}_{12}$ and $\vec{R}_{43}$, these conditions lead to,

$\left[\begin{array}{l}R_{12}^{x} \sin \beta_{12}=R_{12}^{y} \cos \beta_{12} \\ R_{43}^{x} \sin \beta_{43}=R_{43}^{y} \cos \beta_{43}\end{array}\right.$

*Equation (13) is the same for the three cases $A$, B and $C$. i. 
Solving equations (7) for the reactions $R_{12}^{X}, R_{2}^{Y}, R_{43}^{X}, R_{43}^{Y}$ in terms of the accelerations $\left(\ddot{X}_{2}, \ddot{Y}_{2}, \ddot{X}_{3}, \ddot{Y}_{3}, \ddot{Y}_{3}\right)$ and substituting into the results the values of these accelerations using equations (6) and (8) and substituting: the resulting values of these reactions into equations (13) and re-arranging leads to,

$$
\begin{aligned}
\vdots \quad a_{1} \ddot{B}_{12}+a_{2} \ddot{B}_{43} & =a_{3} \\
b_{1} \ddot{\beta}_{12}+b_{2} \ddot{B}_{43} & =b_{3}
\end{aligned}
$$

$\vdots$

The angular accelerations of the clearance angles $\left(\beta_{12}, \beta_{43}\right)$ are obtained from equation (14) as,

$$
\begin{aligned}
\vdots & \ddot{\beta}_{12}=\left(a_{3} b_{2}-a_{2} b_{3}\right) /\left(a_{1} b_{2}-a_{2} b_{1}\right) \\
\ddot{\beta}_{43} & =\left(a_{1} b_{3}-a_{3} b_{1}\right) /\left(a_{1} b_{2}-a_{2} b_{1}\right)
\end{aligned}
$$

Once, the values of $\left(\beta_{12}, \beta_{43}\right),\left(\dot{\beta}_{12}, \dot{\beta}_{43}\right)$ and $\left(\ddot{\beta}_{12}, \ddot{\beta}_{43}\right)$ are evaluated, equa: : tions (2) to (8) are used to evaluate follower motion, reaction forces and torque.

\section{$\because$ Case B: Contact with Cam Noze}

Figure 3 shows the contact at any instant during this period, where

$$
\left(\frac{\pi}{2}-\gamma+\alpha\right) \leqslant \theta_{2} \leqslant\left(\frac{\pi}{2}+y-\alpha\right)
$$

Froin figure 3 the angle $x$, and its derivatives can be written as,

$$
\begin{aligned}
& \lambda= \sin ^{-1}\left[\left(\Delta_{12} \cos \beta_{12}+d \cos \theta_{2}-\Delta_{43} \cos \beta_{43}\right) /\left(r+r_{n}\right)\right] \\
& \lambda=\left(-\Delta_{12} \dot{\beta}_{12} \sin \beta_{12}-d \omega_{2} \sin \theta_{2}+\Delta_{43} \dot{\beta}_{43} \sin \beta_{43}\right) /\left(r+r_{n}\right) \cos \lambda \\
& \lambda=\left\{\left[\Delta_{43}\left(\ddot{\beta}_{43} \sin \beta_{43}+\dot{\beta}_{43}^{2} \cos \beta_{43}\right)-\Delta_{12}\left(\ddot{\beta}_{12} \sin \beta_{12}+\dot{\beta}_{12}^{2} \cos \beta_{12}\right)\right\}\right. \\
&\left.\left.-d \omega_{2}^{2} \cos \theta_{2}\right] /\left(r+r_{n}\right) \cos \lambda\right\}+\dot{\lambda}^{2} \tan \lambda
\end{aligned}
$$

The follower displacement, velocity and accelerations are,

$$
\begin{aligned}
& \gamma_{4}=\Delta_{12} \sin \beta_{12}+d \sin \theta_{2}+\left(r+r_{n}\right) \cos \lambda-\Delta_{43} \sin \beta_{43}-\left(r+r_{b}\right) \\
& \dot{\gamma}_{4}=\Delta_{12} \dot{\beta}_{12} \cos \beta_{12}+d \omega_{2} \cos \theta_{2}-\left(r+r_{n}\right) \dot{\lambda} \sin \lambda-\Delta_{43} \dot{\beta}_{43} \cos \beta_{43}
\end{aligned}
$$




$$
\begin{aligned}
\ddot{\gamma}_{4}= & \Delta_{12}\left(\ddot{\beta}_{12} \cos \beta_{12}-\dot{\beta}_{12}^{2} \sin \beta_{12}\right)-\Delta_{43}\left(\ddot{\beta}_{43} \cos \beta_{43}-\dot{\beta}_{43}^{2} \sin \beta_{43}\right) \\
& -d \omega_{2}^{2} \sin \theta_{2}-\left(r+r_{n}\right)\left(\ddot{\lambda} \sin \lambda+\dot{\lambda}^{2} \cos \lambda\right)
\end{aligned}
$$

Using D'Alembert principle, the dynamic equations of cam, roller and follower are,

$$
\begin{array}{ll}
\vdots & R_{12}^{X}+R_{32} \sin \lambda=m_{2} \ddot{X}_{2} \\
& -R_{12}^{y}-R_{32} \cos \lambda=m_{2} \ddot{Y}_{2} \\
\vdots & -R_{43}^{X}+R_{32} \sin \lambda=m_{3} \ddot{X}_{3} \\
& R_{43}^{y}-R_{32} \cos \lambda=m_{3} \ddot{Y}_{3} \\
\vdots & m_{4} \ddot{Y}_{4}+C \dot{Y}_{4}+k Y_{4}=R_{43}^{y}-F_{0} \\
& T_{2}=m_{2} S\left(\ddot{Y}_{2} \cos \theta_{2}-\ddot{X}_{2} \sin \theta_{2}\right)+R_{32} d \cos \left(\theta_{2}-\lambda\right)
\end{array}
$$

\section{: Cllearance Angles}

The follower displacement $\mathrm{Y}_{4}$ is given by equation (20). From figure 3, : the follower velocity $\dot{Y}_{1}$ can be written as,

$$
\dot{y}_{4}=\omega_{2} \cdot \overline{0_{12} \mathrm{O}_{42}}
$$

¿ where,

$$
\begin{aligned}
\sigma \frac{\sigma_{42}}{\sigma_{12}} & =\left(\Delta_{12} \sin \beta_{12}+d \sin \theta_{2}\right) \tan \lambda+\left(r+r_{n}\right) \sin \lambda \\
& +\Delta_{43} \cos \beta_{43}
\end{aligned}
$$

Since the variations of $\mathrm{Y}_{4}$ and $\dot{\mathrm{Y}}_{4}$ due to clearance effect are negligible, these conditions lead to,

$$
\begin{aligned}
& f_{1}=\left(\sin \beta_{12}-\xi_{1} \cos \beta_{12}\right)-\Delta\left(\sin \beta_{43}-\xi_{1} \cos \beta_{43}\right)=0 \\
& f_{2}=\xi_{1} \sin \beta_{12}+\left(1+\xi_{2}\right) \cos \beta_{12}-\Delta \xi_{2} \cos \beta_{43}=0
\end{aligned}
$$

$$
\text { where, } \begin{aligned}
\xi_{1} & =\beta / \sqrt{1-\beta^{2}} \\
\xi_{2} & =n \sin \theta_{2} /\left(1-\beta_{2}^{2}\right)^{3 / 2} \\
\beta & =n \cos \theta_{2}
\end{aligned}
$$

and

$$
n=d /\left(r+r_{n}\right)
$$

: Equations (24) are solved for the clearance angles $\left(\beta_{12}, \beta_{43}\right)$ using Newton Raphson's method. The derivatives of equation (24) gives,

$$
\quad \quad \underline{A} \cdot \stackrel{\dot{B}}{B}=\vec{v}
$$

where, 
Equation (25) is used to evaluate $\left(\dot{\beta}_{12}, \dot{\beta}_{43}\right)$.

: Solving equations (2I) for the reactions $R_{12}^{\mathrm{X}}, \mathrm{R}_{12}^{\mathrm{Y}}, \mathrm{R}_{43}^{\mathrm{X}}, \mathrm{R}_{43}^{\mathrm{Y}}$ in terms of : the accelerations $\left(\ddot{X}_{2}, \ddot{Y}_{2}, \ddot{X}_{3}, \ddot{Y}_{3}, \ddot{Y}_{4}\right)$ and substituting into the results the values of these accelerations using equations (20) and (8) and substituting : the resulting values of these reactions into equations (13) and re-arrang-
ing leads to,

$$
\begin{aligned}
& \alpha_{12} \ddot{\beta}_{12}+\alpha_{2} \ddot{\beta}_{43} & =\alpha_{3} \\
\vdots & \gamma_{1} \ddot{\beta}_{12}+\gamma_{2} \ddot{\beta}_{43} & =\gamma_{3}
\end{aligned}
$$

$\ddot{B}_{12}$ and $\ddot{\beta}_{43}$ are obtained as,

$$
\begin{aligned}
& \ddot{\beta}_{12}=\left(\alpha_{3} \gamma_{2}-\alpha_{2} \gamma_{3}\right) /\left(\alpha_{1} \gamma_{2}-\alpha_{2} \gamma_{1}\right) \\
& \ddot{\beta}_{43}=\left(\alpha_{1} \gamma_{3}-\alpha_{3} \gamma_{1}\right) /\left(\alpha_{1} \gamma_{2}-\alpha_{2} \gamma_{1}\right)
\end{aligned}
$$

Once, the values of $\left(\beta_{12}, \beta_{43}\right),\left(\dot{\beta}_{12}, \dot{\beta}_{43}\right)$ and $\left(\ddot{\beta}_{12}, \ddot{\beta}_{43}\right)$ are evaluated, equations (17) to (2I) and equation (8) are used to evaluate follower motion, : reaction forces and torque.

Case C: Contact with the Flat Flank During Downward Motion of the Follower

Figure 4 shows the contact at any instant during this period, where,

$$
(\pi / 2+\gamma-\alpha)<\theta_{2} \leqslant(\pi / 2+\gamma)
$$

Similar to section (A), the following equations can be written. 


$$
\begin{aligned}
& r_{1}=\left(r+r_{b}\right)\left(1-\cot \gamma \tan \theta_{2}^{\prime \prime}\right) \\
& \dot{r}_{1}=\left(r+r_{b}\right) \omega_{2} \cot \gamma \sec ^{2} \theta_{2}^{\prime \prime} \\
& \left.\ddot{r}_{1}=-2\left(r+r_{b}\right) \omega_{2}^{2} \cot \gamma \sec ^{2} \theta_{2}^{\prime \prime} \tan \theta_{2}^{\prime \prime}\right\} \\
& q=-D \cos \theta_{2}=-\Delta_{43} \cos \beta_{43}+r_{1} \cos \left(\theta_{2}-\gamma\right)+\Delta_{12} \cos \beta_{12} \\
& \dot{q}=\Delta_{43} \dot{\beta}_{43} \sin \beta_{43}+\dot{r}_{1} \cos \left(\theta_{2}-\gamma\right)-r_{1} \omega_{2} \sin \left(\theta_{2}-\gamma\right)-\Delta_{12} \dot{\beta}_{12} \sin \beta_{12} \\
& \ddot{q}=\Delta_{43}\left(\ddot{\beta}_{43} \sin \beta_{43}+\dot{\beta}_{43}^{2} \cos \beta_{43}\right)-\Delta_{12}\left(\ddot{\beta}_{12} \sin \beta_{12}+\dot{B}_{12}^{2} \cos \beta_{12}\right) \\
& +\left[\left(\ddot{r}_{1}-r_{1} \omega_{2}^{2}\right) \cos \left(\theta_{2}-\gamma\right)-2 \dot{r}_{1} \omega_{2} \sin \left(\theta_{2}-\gamma\right)\right]
\end{aligned}
$$

where,

$$
\begin{aligned}
& \theta_{2}^{\prime \prime}=\left(\gamma-\theta_{2}+\pi / 2\right) \\
& Y_{4}=\Delta_{12} \sin B_{12}-q \tan \theta_{2}+r_{1} \sin \left(\theta_{2}-\gamma\right)-\Delta_{43} \sin B_{43}-\left(r+r_{b}\right) \\
& \dot{Y}_{4}=\frac{\Delta_{12} \dot{\beta}_{12}}{+r_{1} \omega_{2}} \cos \beta_{12}-\frac{\left.\dot{q} \tan \theta_{2}-q \omega_{2}-\gamma\right) \sec ^{2} \theta_{2}+\dot{r}_{1} \sin \left(\theta_{2}-\gamma\right)}{\Delta_{43} \dot{\beta}_{43} \cos \beta_{43}} \\
& \ddot{Y}_{4}=\Delta_{12}\left(\ddot{\beta}_{12} \cos \beta_{12}-\ddot{\beta}_{12}^{2} \sin \beta_{12}\right)-\Delta_{43}\left(\ddot{\beta}_{43} \cos \beta_{43}-\dot{\beta}_{43}^{2} \sin \beta_{43}\right) \\
& -\left(\ddot{q} \tan \theta_{2}+2 \dot{q} \omega_{2} \sec ^{2} \theta_{2}+2 q \omega_{2}^{2} \sec ^{2} \theta_{2} \tan \theta_{2}\right) \\
& +\left[\left(\ddot{r}_{1}-r_{1} \omega_{2}^{2}\right) \sin \left(\theta_{2}-\gamma\right)+2 \dot{r}_{1} \omega_{2} \cos \left(\theta_{2}-\gamma\right)\right] \\
& R_{12}^{x}-R_{32} \cos \left(\theta_{2}-\gamma\right)=m_{2} \ddot{x}_{2} \\
& -R_{12}^{y}-R_{32} \sin \left(\theta_{2}-\gamma\right)=m_{2} \ddot{Y}_{2} \\
& -R_{43}^{X}-R_{32} \cos \left(\theta_{2}-\gamma\right)=m_{3} \ddot{x}_{3} \\
& R_{43}^{y}-R_{32} \sin \left(\theta_{2}-\gamma\right)=m_{3} \ddot{y}_{3} \\
& m_{4} \ddot{Y}_{4}+C \dot{\gamma}_{4}+K Y_{4}=R_{43}^{y}-F_{0} \\
& T_{2}=m_{2} S\left(\ddot{\gamma}_{2} \cos \theta_{2}-\ddot{x}_{2} \sin \theta_{2}\right)+\left(q \sin \gamma / \cos \theta_{2}\right) R_{32}
\end{aligned}
$$

\section{Clearance Angles}

The follower displacement $\mathrm{Y}_{4}$ is given by equation (33). From figure 4, the follower-velocity $\dot{\mathrm{Y}}_{4}$ can be written as,

$$
\dot{\gamma}_{4}=\omega_{2} \cdot \overline{\sigma_{12} \theta_{42}}
$$

where,

$$
\begin{aligned}
& \overline{0_{12} 0_{42}}=\left[\left(\Delta_{12} \sin \beta_{12}-q \cdot \tan \theta_{2}\right) / \tan \left(\theta_{2}-\gamma\right)\right]+r_{1} \cos \left(\theta_{2}-\gamma\right) \\
& \text { L.. } \quad \ldots \quad \ldots-\Delta_{43} \cos \beta_{43} \ldots
\end{aligned}
$$


Since, the varittions of $\mathrm{Y}_{4}$ and $\dot{\mathrm{Y}}_{4}$ due to clearance effect are negligible, :these conditions lead to,

$$
\begin{aligned}
f_{1} & =\sin \left(\beta_{12}-\theta_{2}\right)-\Delta \sin \left(\beta_{43}-\theta_{2}\right)=0 \\
f_{2} & =\sin \left(\beta_{12}-\theta_{2}\right)-\Delta f\left(\theta_{2}\right) \cos \beta_{43}=0
\end{aligned}
$$

where,

$$
f\left(\theta_{2}\right)=-\sin \theta_{2}+\cos \theta_{2} \tan \left(\theta_{2}-\gamma\right)
$$

Equations (37) are solved for the clearance angles $\left(\beta_{12}, \beta_{43}\right)$ using Newton Raphson's method. The derivatives of equation (37) give,

$$
\begin{aligned}
& \dot{\beta}_{43}=\omega_{2}\left[f^{\prime}\left(\theta_{2}\right) \cos \beta_{43}+\cos \left(\beta_{43}-\theta_{2}\right)\right] /\left[f\left(\theta_{2}\right) \sin \beta_{43}+\cos \left(\beta_{43}-\theta_{2}\right)\right] \\
& \vdots \dot{\beta}_{12}=\Delta \xi \dot{\beta}_{43}+(1-\Delta \xi) \omega_{2} \\
& f_{\text {where, }}^{\prime \prime}\left(\theta_{2}\right)=-\cos \theta_{2}-\sin \theta_{2} \tan \left(\theta_{2}-\gamma\right)+\cos \theta_{2} \sec ^{2}\left(\theta_{2}-\gamma\right)
\end{aligned}
$$

: Equations (38) are used to evalute $\left(\dot{\beta}_{12}, \dot{B}_{43}\right)$. Solving equations (34) for. the reactions $R_{I_{2}}^{X}, R_{2}^{Y}, R R_{3}^{X}, R H_{3}$ in terms the accelerations $\left(\ddot{X}_{2}, \ddot{Y}_{2}, \ddot{X}_{3}, \ddot{Y}_{3}, \ddot{Y}_{4}\right)$ and substituting into the results the values of these accelerations using: : equations (33) and (8) and substituting the resulting values of these reactions into equations (13) and rearranging leads to,

$$
\begin{gathered}
\mu_{1} \ddot{\beta}_{12}+\mu_{2} \ddot{\beta}_{43}=\mu_{3} \\
\delta_{1} \ddot{\beta}_{12}+\delta_{2} \ddot{\beta}_{43}=\delta_{3} \\
\left(\ddot{\beta}_{12}, \ddot{\beta}_{43}\right) \text { are obtained as, } \\
\ddot{\beta}_{12}=\left(\mu_{3} \delta_{2}-\mu_{2} \delta_{3}\right) /\left(\mu_{1} \delta_{2}-\mu_{2} \delta_{1}\right) \\
\ddot{\beta}_{33}=\left(\mu_{1} \delta_{3}-\mu_{3} \delta_{1}\right) /\left(\mu_{1} \delta_{2}-\mu_{2} \delta_{1}\right)
\end{gathered}
$$

Once, the values of $\left(\beta_{12}, \beta_{43}\right),\left(\dot{\beta}_{12}, \dot{\beta}_{43}\right)$ and $\left(\ddot{\beta}_{12}, \ddot{\beta}_{43}\right)$ are evaluated, equations (29) to (34) and equation (8) are used to evaluate follower motion, reaction forces and torque.

A Fortran IV computer program is written and used to evaluate the kinematic and dynamic quantities for a cam mechanism with two bearing clearances. Figure 5 shows a flow chart of the computation procedure.

\section{RESULTS AND CONCLUSIONS}

The following input data for a cam mechanism is used.

$r_{b}=0.03388 \mathrm{~m} ., r_{n}=0.0275 \mathrm{~m} ., r=0.01375 \mathrm{~m} ., \quad d=0.01914 \mathrm{~m}$.

$\vdots \mathrm{S}=0.03364 \mathrm{~m} ., \Delta_{12}=100 . \mu \mathrm{m} ., \Delta_{43}=60 . \mu \mathrm{m}$.

$m_{2}=1.8 \mathrm{~kg} \cdot \quad, m_{3}=0.0894 \mathrm{~kg} \cdot, m_{4}=1.139 \mathrm{~kg}$.

$\vdots \mathrm{F}_{\mathrm{O}}=24.2293 \mathrm{~N} ., \mathrm{K}=7787.79 \mathrm{~N} / \mathrm{m}, \mathrm{C}=9.7812 \mathrm{~N} . \mathrm{s} . / \mathrm{m}$.

: The input speed to the driving cam is assumed 60.rad./s. anticlockwise. 
Figures 6 to 12 show the computer analysis results of the cam mechanism. The results are compared with the case when clearance is ignored. There are considerable changes in accelerations, bearing reaction forces and torques due to the effect of bearing clearances. The changes cf bearing reaction forces and torques are mainly due to the changes in accelerations.

Figures 6 and 7 show the variations of clearance angles $\left(\beta_{12}, \beta_{43}\right)$ during the angle of action of the cam. There are changes in $\beta_{j 2}$ in the range of

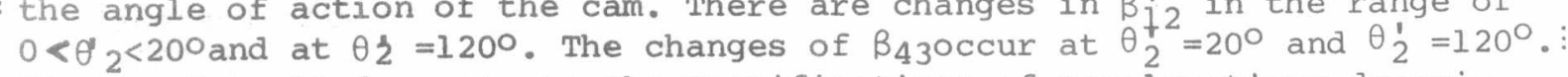
Figures 8 to 12 demonstrate the magnifications of accelerations, dynamic

forces and torques when clearance effect is taken into account. At $\theta_{2}^{\prime}=50$ there is an instant of separation since, the reaction forces change from : : positive to zero and vice-versa.

It is important to note that the magnifications of accelerations forces and : torques in the cam mechanism as affected by revolute clearances essentially depend on clearance sizes and operating speed of the cam shaft. Cam and follower dimensions have a small effect compared to that of clearance size:

: or operating speed.

The results indicated in this paper represent an upper bound of the bearing : reaction forces due to the existence of bearing clearance, since the stiffness and damping of oil films and a part of the system elasticity are not included in the analysis. These effects tend to reduce the values of these : bearing reaction forces.

\section{REFERENCES}

1. Chen,F.Y.,"Analysis and Design of Cam-Driven Mechanisms with Non-linearities", Journal of Eng.for Ind.Trans.ASME.,1972,Vol.95,pp.685-694.

2. Wiederrich,J.L.,Roth,B.,"Dynamic Synthesis of Cams Using Finite Trigonometric Series", Jounal of Eng. for Ind.Trans,ASME, Vol.97, No.1, $1975, \mathrm{pp} .287-293$.

3. Koster,M.P.,"Effect of Flexibility of Driving Shaft on the Dynamic Behavior of a Cam-Mechanism",Journal of Eng.for Ind.Trans,ASME,Vol.97, No. 2,1975, pp.595-602.

4. Chen,F.Y.,Polvanich,N.,"Dynamics of High-Speed Cam-Driven Mechanism, Part 1: Linear System Models",Journal of Eng.for Indu. Trans. ASME, Vol.

: $\quad 97$, No $.3,1975, \mathrm{pp} .769-776$.

5. Chen.F.Y.,Polvanich,N.,"Dynamics of High-Speed Cam-Driven Mechanism, Part 2: Non-Linear System Models",Journal of Eng.for Ind.Trans.ASME,Vol. 97, No.3,1975, pp.777-784.

6. Matthew,G.K.,Tesar,D.,"The Dynamic Synthesis and Analysis of the OneDegree-of-Freedom Model",Journal of Mech,Mach, Theory,Vol.11, No.4,1975,: pp. 247-257.

7. Matthew,G.K.,Tesar,D.,"The Design of Modeled Cam Systems,Part 1:Dynami.C : Synthesis and Chart Design for the Two-Degree-of-Freedom Model",Journal of Eng.for Ind.Trans.ASME,Vol.97, No.4,1975,pp.1175-1180.

: 8. Matthew,G.K., Tesar,D.,"The Design of Modeled Cam Systems,Part II,Minimization of Motion Distortion due to Modelling Errors",Journal of Eng.for Ind. Trans. ASME, Vol . 97 , No .4,1975,pp.1181-1189. 
9. Koster,M.P." "Digital Simulation of the Dynamics of Cam,Followers and Cam Shafts" World Congr.on the Thoery of Mach.and Mech., 1975,pp.969974.

10. Tesar,D. "Matthew,G.K. "Dynamic Distortion in Cam Systems",Journal of Mach.Des.,Vol.48,No.7,1976,pp.186-191.

11. Chen,F。Y., "Dynamic Response of a Cam Actuated Mechanism with Pneumatic Coupling",Journal of Eng.for Ind.Trans. ASME, Vol.99, No.3, 1977, pp. 598603.

12. Sankar,S. Osman,M.O.M. "Dynamic Accuracy of Hybrid Profiling Mechanism in Cam Manufacturing" "Journal of Mechanical Design,Vol.101,No.1,1977, pp. 108-117.

13. Rao,A.C., "Optimum Elastodynamic Synthesis of Cam-Follower Train Using Stochastic-Geometric Programming" "Journal of Mech.Mach.Theory,Vol.15, No.2,1980,pp.127-135.

14. Szakallas,L.E.,Savage,M.,"The Charactrization of Cam-Drive System Wind, Up", Journal of Mech.Des.Trans.ASME,Vol.102, No.2, 1980,pp. 278-285.

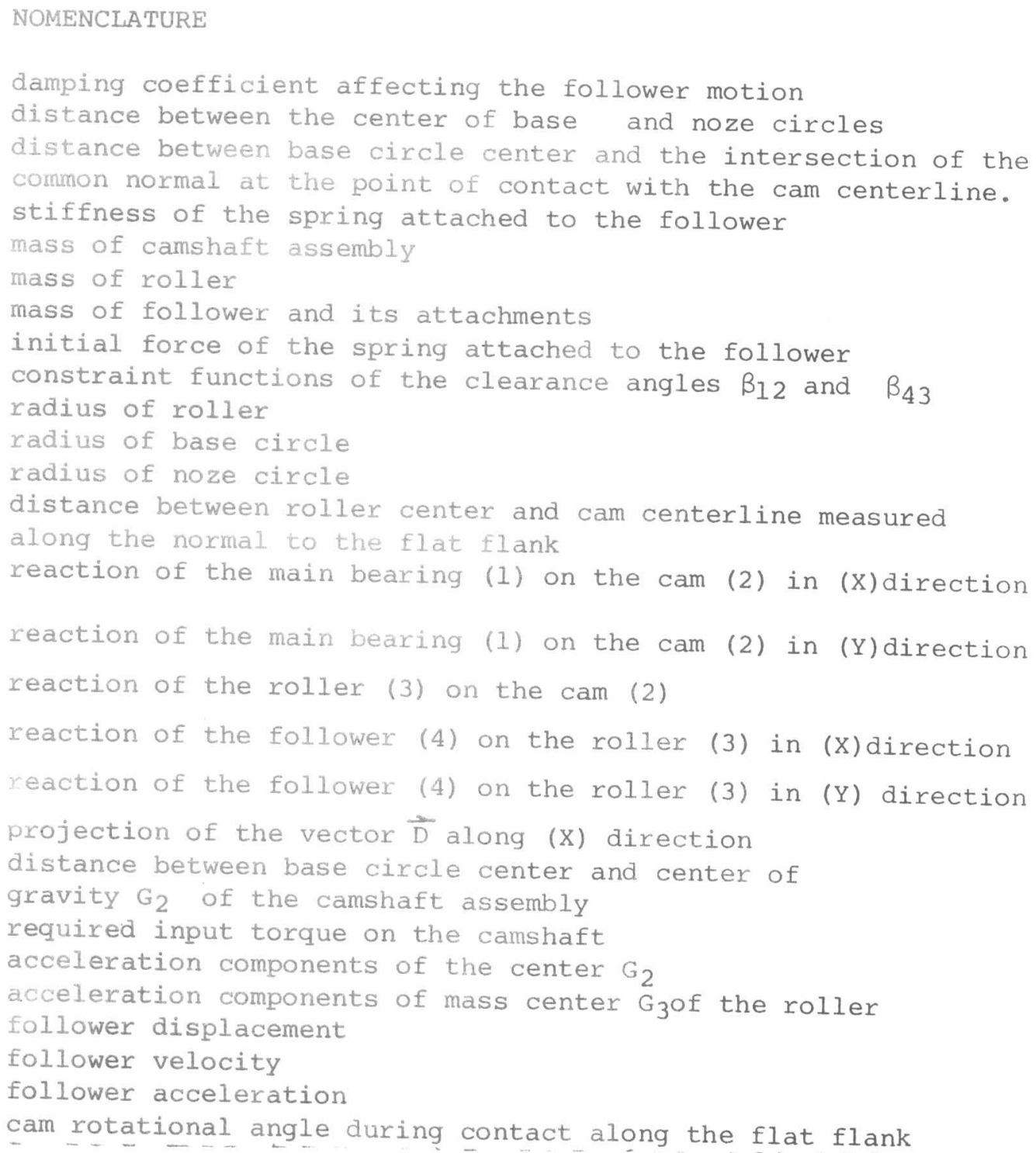


clearance angles at the main bearing and the roller bearing 
$[D Y N-14 \mid 154$

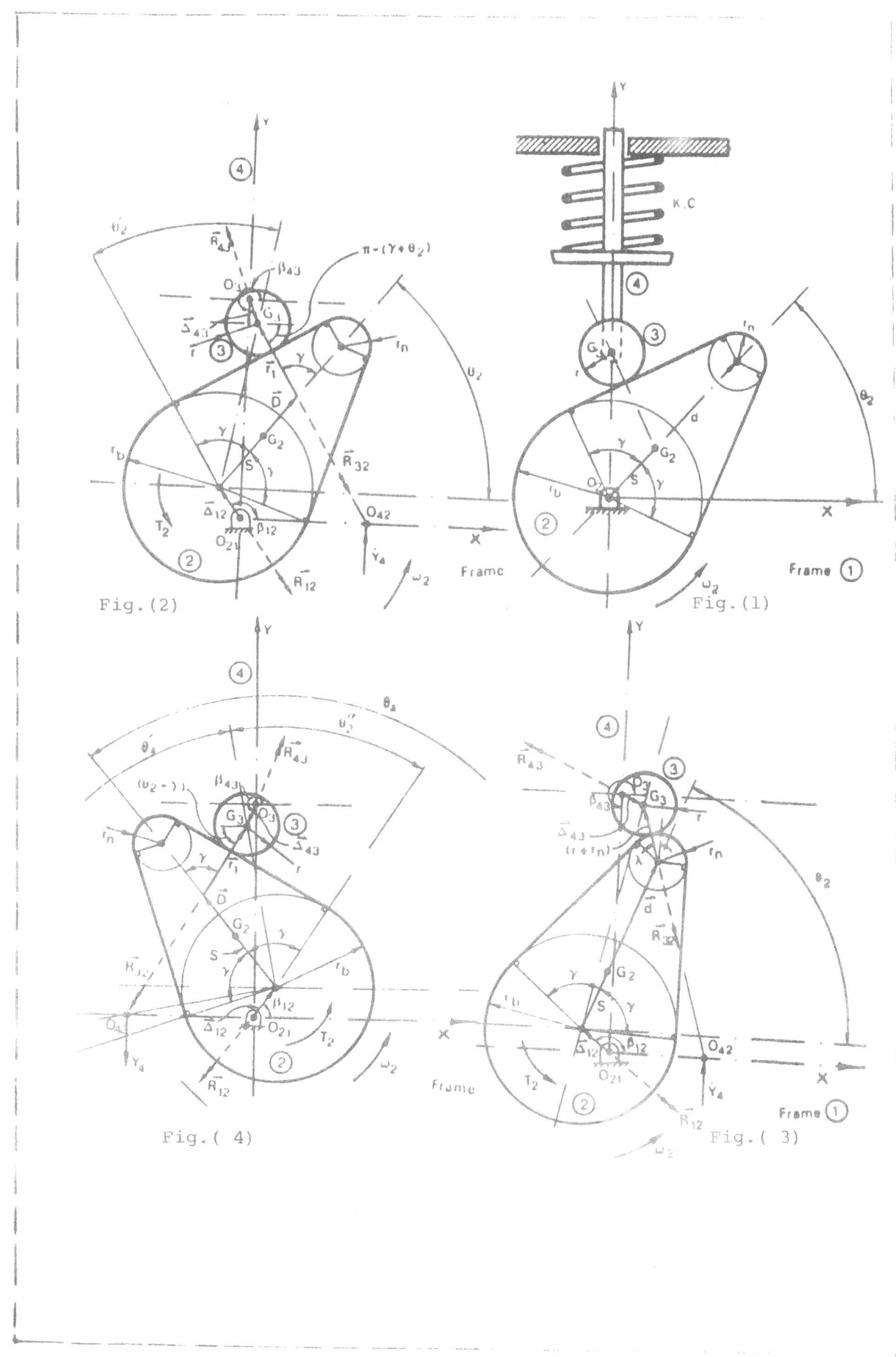




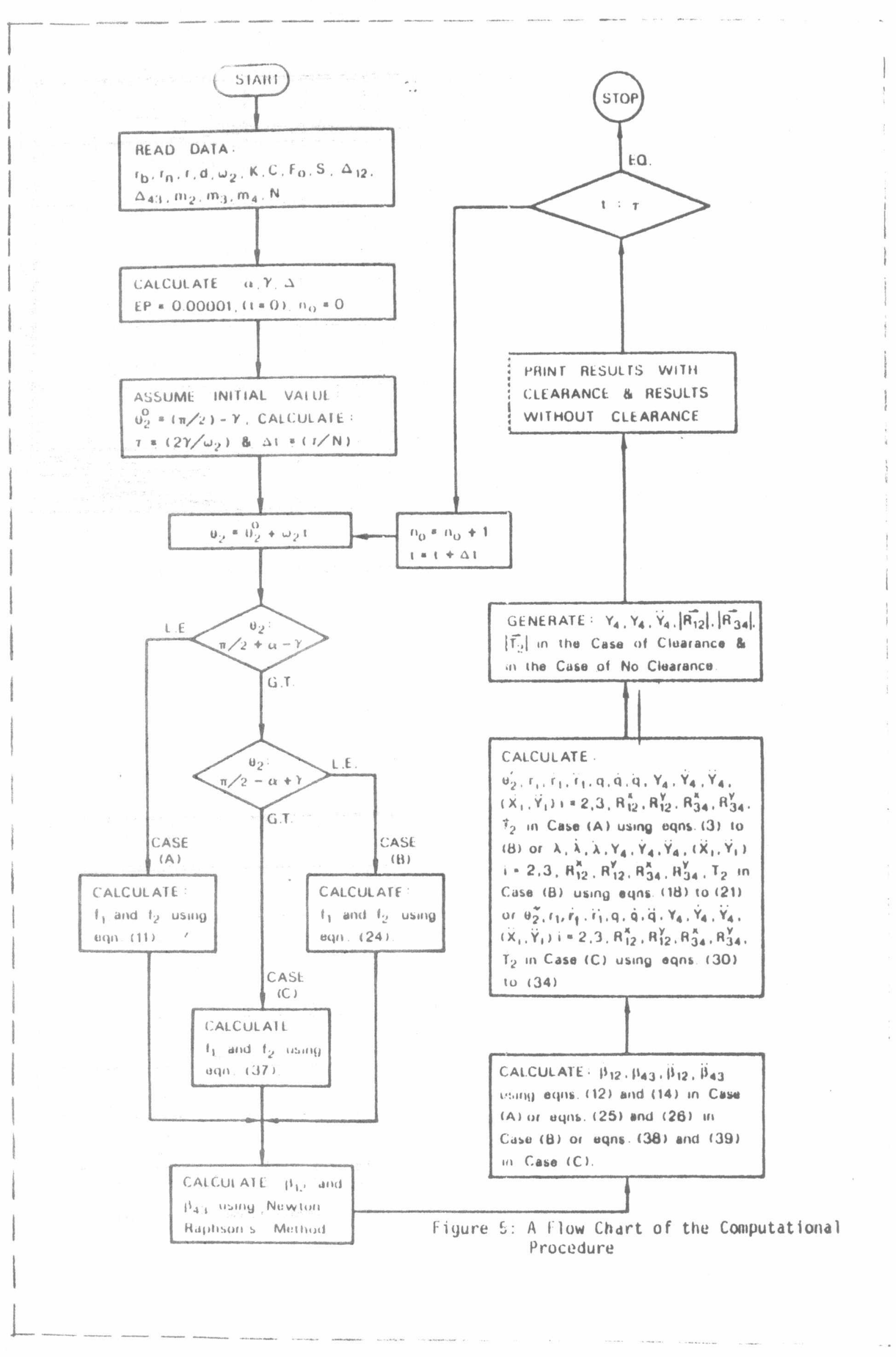




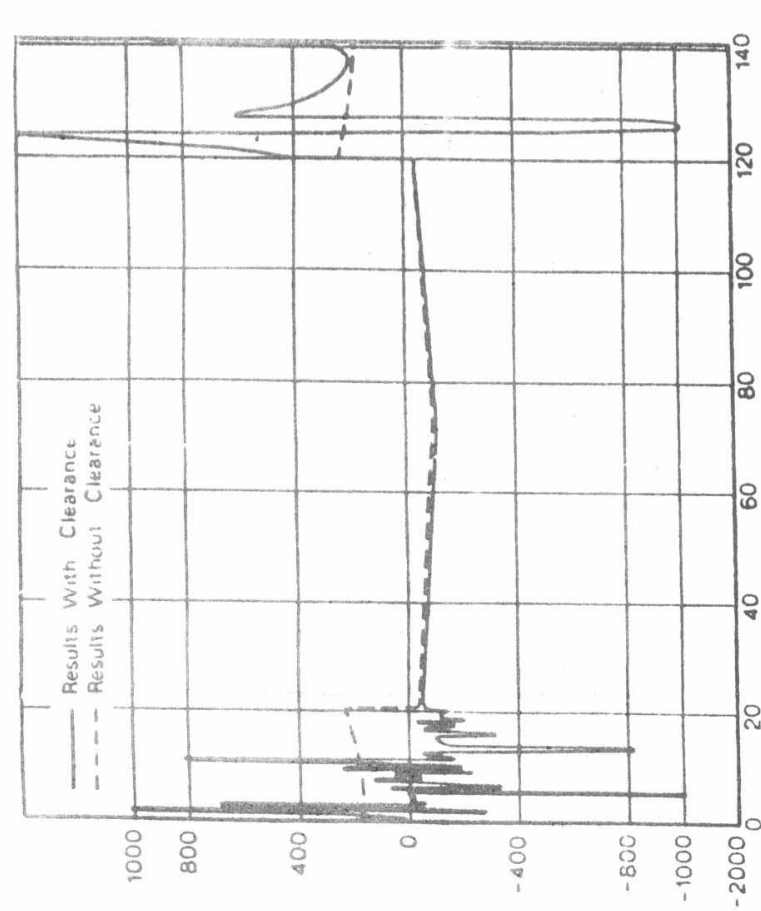

$\left(2^{5 / 41}\right)^{D A}$ a

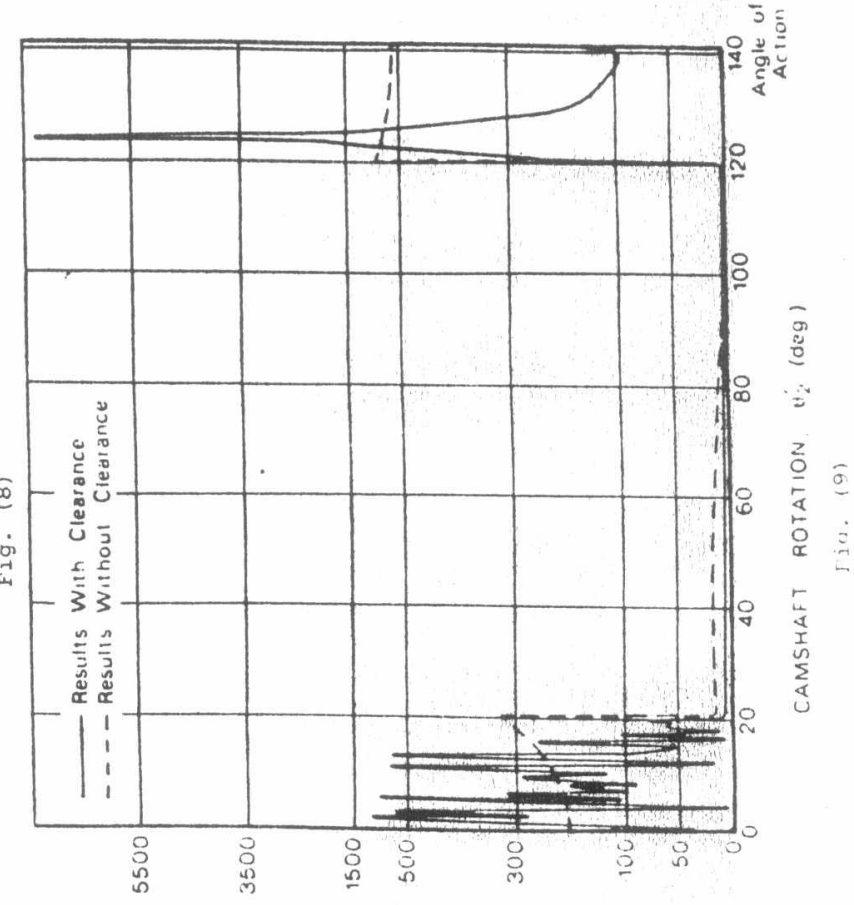

(N) F́t

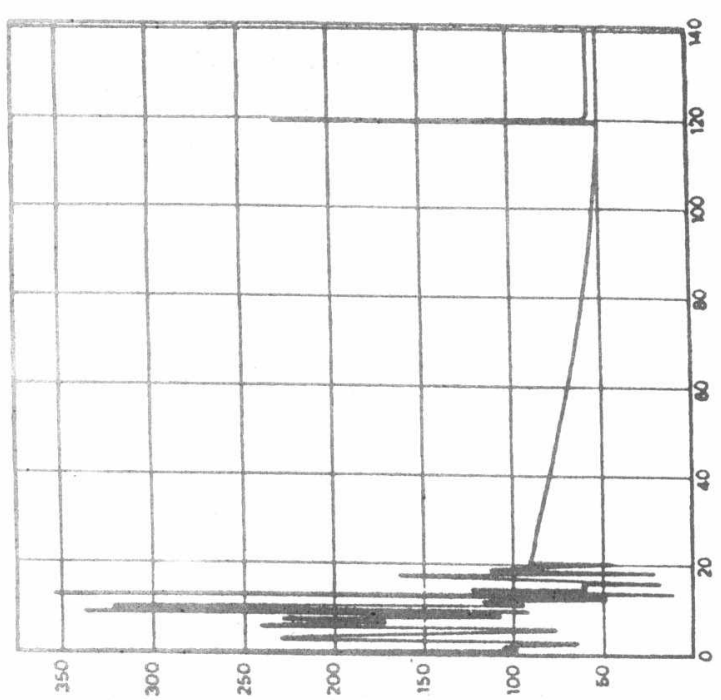

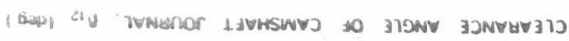

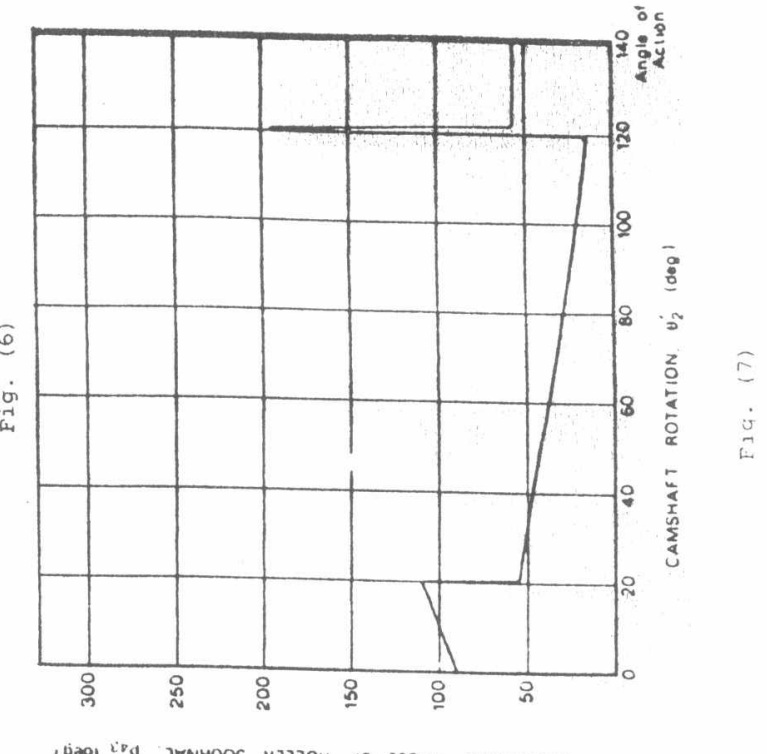

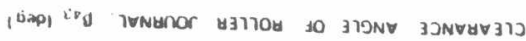




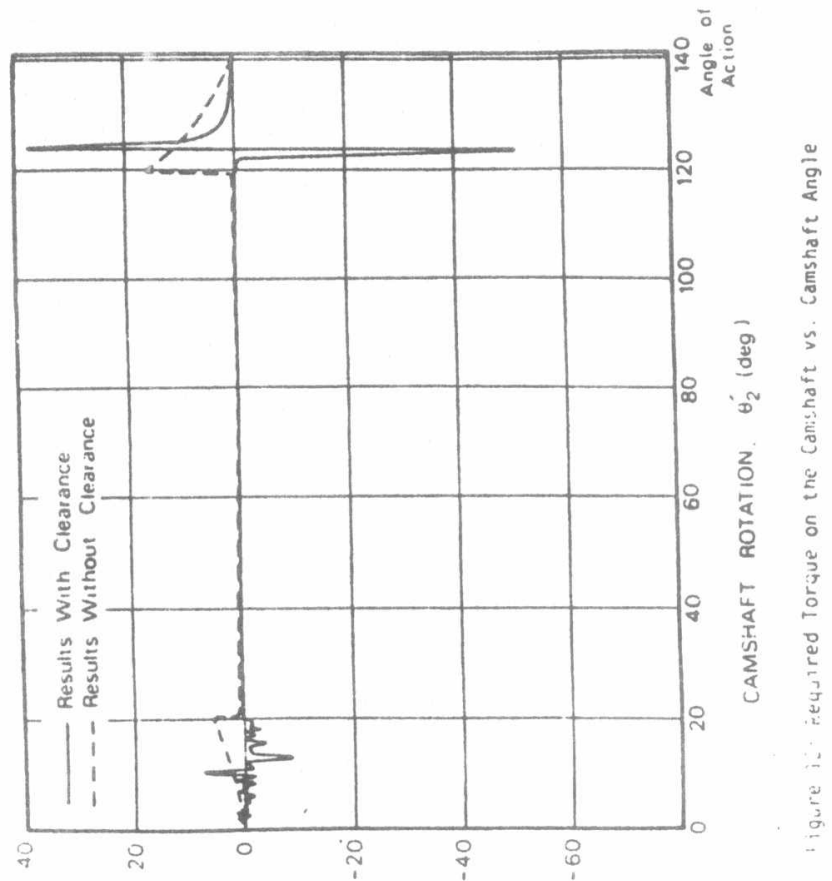

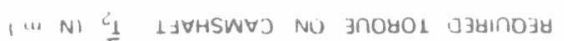
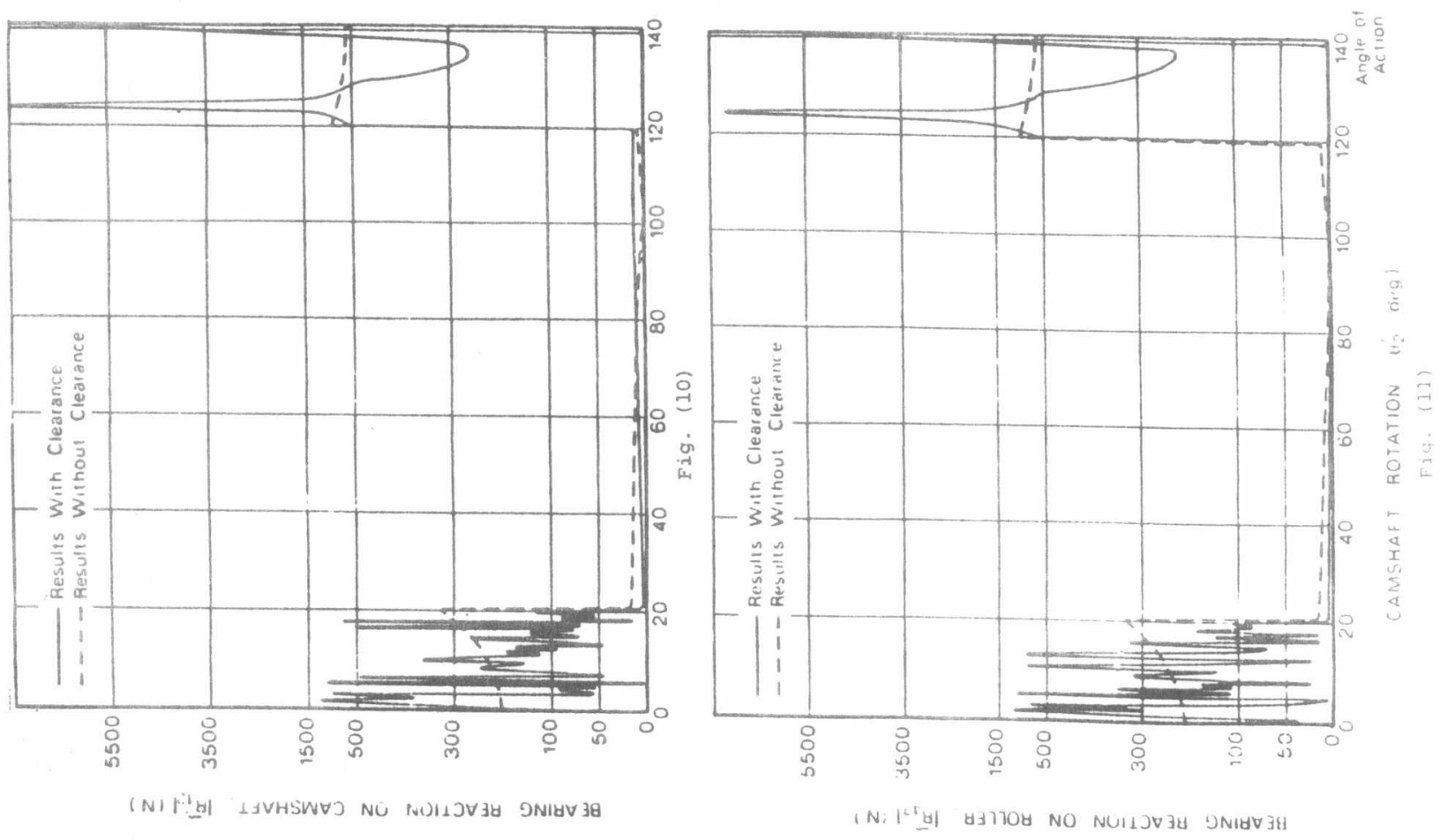
